\title{
Research on Path Optimization of Ant Colony Algorithm Improved Particle Swarm Optimization and Reverse Learning
}

\author{
Shaobo $\mathrm{Li}^{1,3}$, Kangqi $\mathrm{Mu}^{1,}{ }^{*}$, Weimin $\mathrm{Lin}^{2}$, Dong Sun ${ }^{1}$ \\ 1School of Mechanical Engineering, Guizhou University, Guiyang, 550025, China; \\ ${ }^{2}$ Key Laboratory of Advanced Manufacturing Technology, Guizhou University, Guiyang, 550025, \\ China; \\ ${ }^{3}$ Guizhou Provincial Key Laboratory of Public Big Data (Guizhou University), Guiyang, Guizhou \\ 550025, China. \\ *1147834557@qq.com
}

\begin{abstract}
Keywords: Ant colony algorithm; Particle swarm optimization; reverse learning strategy; Pheromone update.
\end{abstract}

\begin{abstract}
Aiming at the difficulty of determining the key parameters when applying ant colony optimization algorithm (ACO) to traveling salesman problem, we propose an improved particle swarm optimization (PSO) algorithm for adaptive parameter acquisition. Because repeated calls to ACO will increase the cost of computing and get the local optimal solution easily, the number of single ACO iterations is reduced, and the update of the pheromone is determined by the fitness function. After each call to ACO, the pheromone is not adjusted. In order to get better quality parameters of PSO, the reverse learning strategy is applied to PSO, and the speed of optimization is improved. The effectiveness of the algorithm is proved by the simulation experiment.
\end{abstract}

\section{Introduction}

The problem of path planning has attracted wide attention since it was put forward in 1950s. In the logistics industry, with the continuous change of the types of logistics transportation products, it puts forward more and higher requirements for the logistics and transportation industry. The intelligent transportation mode is gradually integrating into the logistics industry, based on large data analysis, it is the development direction of the present transportation to integrate the heuristic intelligent algorithm into the complex and repetitive handling work, so as to realize the logistics under the data. The transport network of logistics is responsible for a wide variety of commodity exchange, when we choose the route of transportation, we must consider multiple factors comprehensively, formulate reasonable transportation programs and respond to customer needs quickly, so as to enhance customer satisfaction, reduce transportation costs and improve delivery quality. The application of intelligent algorithm to the solution of NP problem is a problem that many scholars have paid attention to in recent years. On the one hand, the way to improve the solution of the problem, on the other hand, is constantly changing the way people live. The starting point of the ant colony algorithm is to solve the traveling salesman problem, using the pheromone exchange ability of ants to plan a shortest path as much as possible. This paper [1] applied the smooth ant colony algorithm to the robot path planning, which solved the problem of the larger angle of the route angle. But the disadvantage of the algorithm is premature convergence and local optimum, so many scholars have carried out a variety of improvement methods. The ant system proposed by Dorito [2] and the maximum and minimum ant system [3], etc. Huang [4] takes the time window of fruit and vegetable quality as a constraint and uses the improved ant colony algorithm to solve the distribution path modeling. Article [5] provides the ACS model in beta, $\mathrm{P}$ and Q0 optimization method and the parameter [6] in the algorithm of alpha and beta, Rho optimization, has achieved good results, but at the cost of larger calculation. You [7] proposes a dynamic search induction operator and solves the problem of path optimization of mobile robot in complex environment by improving ant colony algorithm. 
The reverse learning method is proposed by Tizhoosh, reverse population is produced by reverse learning strategies, then screening current and reverse populations to avoid the omission of the global optimal individual as far as possible. The accuracy of the initial guess value is closely related to the iterative optimization of the algorithm, Tizhoosh thinks. Therefore, taking into account the accuracy of initial guess is the main factor in reverse learning. Wang has achieved good results through Appling strategy of reverse to search optimization algorithms [8]. PSO is an analogous bird's foraging behavior, and the search space is simulated as a bird's flight space. The algorithm has good generality and strong global optimization ability, but its optimization ability is largely related to the initial solution. If the initial solution is good, the optimization rate of the particle will be greatly accelerated, conversely, the optimization ability will be weakened. Chen [9] adopts improved PSO - ACO algorithm to solve the problem of multi target and bilateral matching and improves the optimization speed of the algorithm. So, the combination of the reverse learning strategy [10] and the particle swarm optimization algorithm enhance the convergence rate of particle swarm and reduce the cost of calculation and improving the calculation rate. In the experiment, the results of the particle swarm algorithm are imported to the ant colony algorithm, at the same time, the update of pheromone in the path of ant colony optimization is improved. The number of iterations of the single ant colony algorithm is reduced, and the improved method is verified by the experiment. The calculated results have achieved the expected effect.

\section{Algorithm Theory}

\subsection{Particle Swarm Optimization.}

The particle swarm algorithm is derived from the simulation of foraging behavior in the migratory activities of birds, and the initial solution is random. Then the optimization iteration is carried out, and the optimal solution is finally found in the solution space. In PSO, particles are promoted by cognition and learning to the surrounding particles. Self-aligning, finally find the optimal solution. The velocity variable of the particle in the $\mathrm{D}$ dimensional space is $V_{i}=\left(V_{i 1}, V_{i 2} \ldots, V_{i n}\right)$, the velocity variable is $X_{i}=\left(X_{i 1}, X_{i 2} \ldots, X_{i n}\right)$, the position of the particle is updated by the local optimal value Pub and the global optimal value GB in the iterative optimization process. This way of imparting the high-quality genes can make the particle take into account the extension of the particle in the process of optimization. Not only the local optimal solution particles are taken into account, but also the global particles are considered, and the self-learning and mutual learning are constantly carried out, and the optimal solution is finally found. The updating formula of particle position and velocity is as follows:

$$
\begin{gathered}
V_{i}(k+1)=\omega V_{i}(k)+C_{1} r_{1}\left(P_{b}-X_{i}(k)\right)+C_{2} r_{2}\left(G_{b}-X_{i}(k)\right) \\
X_{i}(k+1)=X_{i}(k)+V_{i}(k+1)
\end{gathered}
$$

Where $\omega$ is inertia weight, which controls the linear motion of a particle, the value between 0.4 0.9 and search the optimal solution in the global range. $C_{1}, C_{2}$ is learning factor, which controls the ability of particles to learn from themselves and communicate with each other to get better $P_{b}$ and $G_{b} . R_{1}$ And $R_{2}$ are random numbers, the value between $0 \sim 1$. The upper and lower limit of the velocity of a particle is given, $V_{i} \in\left[-V_{\max }, V_{\max }\right]$. Iterative termination conditions are determined by maximum iterations or precision thresholds.

\subsection{Reverse Learning Strategy.}

Considering the evolutionary speed of the algorithm is related to the initial estimate, in the initialization stage, calculating the current value, at the same time, calculating the reverse value. Finally, get the best value by comparing the two results [11-12]. Briefly introduce the following concepts for describing easily.

Define $1 X \in[a, b] \quad X$ is real number, and $\dot{X}$ is the reverse number of $X$ 
Extend to high dimensional space:

$$
\dot{X}=a+b-X
$$

Define $2 P=\left(y_{1}, y_{2} \ldots y_{i} \ldots y_{D}\right), y_{i} \in\left[a_{i}, b_{i}\right], i \in\{1,2 \ldots, D\}$, the point of the reverse number is $\dot{P}=\left(\dot{y}_{1}, \dot{y}_{2} \ldots, \dot{y_{D}},\right), \dot{y}_{i}=\left(a_{i}+b_{i}-y_{i}\right)$. Using $g$ as the fitness function of a decision point. If $\dot{g}(\dot{P}) \leq g(P)$, Regard $P$ as merit point, conversely, Regard $\dot{P}$ as merit point. In this way, the best particle of $M$ fitness value is chosen from the population as the initial population.

Process implementation of particle swarm optimization with reverse strategy:

Algorithm 1: Particle swarm optimization with reverse strategy (PSO - RS)

Input: Initialization population $P(S)$, Inertia weight $\omega, C_{1}, C_{2}$;

Output: particle's position $X_{i}$, speed $V_{i}$, position $X_{i}$ and speed $V_{i}$; inverse solution of $i$ particle; fitness value $g_{i}$ and its inverse solution fitness value $\dot{g}_{i}$.

$S$ Is the population number,

Then, Compare $g_{i}$ and $\dot{g}_{i}$, if $g_{i}>\dot{g}_{i}$, then $X_{i}=\dot{X}_{i}, g_{i}=\dot{g_{i}}$;

Finally, selecting $S$ particles to form the initial population parameters as the parameters of the ant colony algorithm

\section{Mathematic Model}

Modeling the case of traditional Chinese medicine distribution. A distribution company carries out the delivery of the goods for the various medical units, consider the special properties of medicine, the special needs and the geographical location of each demand unit and the single vehicle weight and other factors, arrange the distribution route reasonably, reduce the delivery cost to the lowest, and establish the following constraints.

1). each unit has only one vehicle to be delivered, and only one car can be completed.

2). the vehicle starts from the general company and returns to the general company after the delivery. The total volume of cargo is not more than the load of the vehicle.

3 ). the demand for each unit is not greater than the total number of vehicles.

4). each car has the same load.

Define variable:

$l_{i k}=1$ or 0 the $i$ point is delivered by $k$ is 1 , otherwise 0 .

$m_{i j k}=1$ or 0 the $k$ car runs from point $i$ to $j$ is 1 , otherwise 0

Then the target function can be expressed as

$$
\begin{gathered}
\min z=\sum_{i=0}^{n} \sum_{j=0}^{n} \sum_{k=1}^{m} C_{i j} C_{i j k} \\
\sum_{i} g_{i} l_{i k} \leq q \quad \forall k \\
\sum_{i} l_{i k}=1 \quad i=1 \ldots, n \\
\sum_{j} X_{k i j k}=y_{k j} \quad j=0,1 \ldots, n ; \forall k \\
\sum_{i} X_{k i j k}=y_{k i} \quad i=0,1 \ldots, n ; \forall k \\
m=\left(m_{i j k}\right) \in D
\end{gathered}
$$


$C_{i j}$ Is transportation cost from $i$ to $j$ ? $g_{i}$ Is the demand for $i, q$ is vehicle load. $X_{k i j k}$ Is the amount of goods of vehicle $k$ along a path? $\sum_{i} g_{i} l_{i k}$ Indicate single cycle carrying capacity is not greater than load; $\sum_{i} l_{i k}$ indicate the $i$ point is delivered by $k$.

\section{Improvement of Ant Colony Algorithm and Process Implementation}

The intelligent evolutionary algorithm proposed by Dorito for the foraging behavior of simulated ants is essentially parallel algorithm, finding the best solution by collaboration among individuals. The algorithm has the characteristics of heuristic search in distributed computing. But it is easy to get into the local optimal and the search time is too long. Ant colony algorithm is used to solve the traveling salesman problem, and it is the first problem to solve the problem, in the process of evolution, the ant is a traveler. From a point, go through all the target points and only be able to visit once, then return to the starting point, this process builds the path. The initial state of the ant individual is independent from each other during the process of evolution, the ants walk through each point in turn, ant transfer probability is influenced pheromone, finally find an optimal path. In this paper, ACO model is taken as an example, $P_{i j}^{k}(t)$ indicates the selection probability of ants from the city $i$ to $j$.

$$
P_{i j}^{k}(t)=\left\{\begin{array}{c}
\frac{\tau_{i j}^{\alpha}(t) \varphi_{i j}^{\beta}(t)}{\sum_{s \in \text { allowed }_{k}} \tau_{i s}^{\alpha}(t)}, j \in \text { allowed }_{k} ; \\
0, \text { otherwise. }
\end{array}\right\}
$$

(1). Initialization parameters. Ant number: $m$; City number: $n$. Number of iterations: Max; Initial path pheromone: $\Delta_{i j}=0$, Pheromone from $i$ to $j . \varphi_{i j}(t)$ Is heuristic function, the expectation of ants from $i$ to $j$, the general value of the reciprocal of the distance between two cities? A collection of cities to be visited: allowed $_{k}$.

The importance of $\alpha$ as $\tau$, the importance of $\beta$ as $\varphi$

(2) The updating of pheromone uses the combination of local pheromone updating and global pheromone updating, the principle of combining global asynchrony with elite strategy. When an ant begins to visit from a city, after all the cities are seen as a week of travel, a set of feasible solutions is formed at this time. The pheromone is released on the path of the ant, and it is determined to get a stronger solution based on the current fitness function, If the better solution, then update the pheromone. Conversely, no pheromones are updated. Pheromone updating formula is:

$$
\begin{gathered}
\tau_{i j}(t+1)=\tau_{i j}(t)+\Delta \tau_{i j}(t) \\
\Delta \tau_{i j}(t)=\left\{\begin{array}{c}
\frac{Q}{G_{k}},[i, j] \text { in the best } \\
0, \text { otherwise }
\end{array}\right\}
\end{gathered}
$$

$G_{k}$ Is the shortest distance, $\Delta \tau_{i j}(t)$ is the increment of $[i, j]$ pheromone on the path after each cycle. Total pheromone: $Q G_{k}$ Is the shortest path of the sub cycle of ant $K$. Ants constantly search the city points to build the optimal solution. The pheromone on the path is constantly updated with the quality solution, and the pheromone on the path passed by the global optimal solution is strengthened, and the terminating condition is finally reached (Iterative termination conditions are determined by maximum iterations or precision thresholds).

$\alpha, \beta, \rho$ Is used as the parameter of particle swarm optimization and the results are grouped into ant colony algorithm, the excellent degree of particle swarm optimization results is evaluated by ant colony algorithm. In order to take into account, the speed of the algorithm and the quality of the 
solution, the number of iterations for each call of the ant colony algorithm is 10 times. An ant colony algorithm is called once per switching parameter. ACS realization:

Algorithm 2: Ant colony optimization with Particle swarm optimization (ACO - PSO)

Input: $\alpha, \beta, \rho$, the initial pheromone is 0 , Other parameters [13-14];

Output: $\sigma$ or calculate the number of iterations;

Switching $\alpha, \beta, \rho$, the pheromone is not initialized, and continues the next round of iterative loops.

Repeat the above process until the number of iterations is reached or the two adjacent optimal solutions are less than $\sigma$, and the cycle ends.

\section{Simulation Experiment and Example Analysis}

To test the performance of the proposed algorithm in this article, the experimental process is based on Visual C+ +2010 and Mat lab R2013a. The measured data are the same models purchased at the same batch. On the PC computer, it is configured to be: AMD TM II X4820(2.59GHz) CPU, $2 \mathrm{~GB}$ RAM.

The application case is 1 main stations, 8 demand points, 2 distribution vehicles, load $8 \mathrm{t}$. The distance between the station and the demand point is shown in Table 2. In order to avoid the randomness of the running results, the selected parameters of each group are run 10 times separately, as shown in the result. In the experiment, each car started from the distribution station, itinerant, and finally came back to the starting point. The following graph is the selection of the optimal solution for the average distance and the shortest distance. Table.1 operation result:

Table 1 . The combination of high quality parameters with reverse learning

\begin{tabular}{ccccc}
\hline Group number & $\alpha$ & $\beta$ & $\rho$ & Shortest path \\
\hline 1 & 1.3012 & 4.6251 & 0.4572 & 518.809 \\
2 & 1.1033 & 4.6205 & 0.5164 & 519.1568 \\
3 & 2.5842 & 4.7013 & 0.4752 & 518.9901 \\
4 & 3.5108 & 4.6641 & 0.4809 & 518.7612 \\
5 & 1.0856 & 4.7002 & 0.3918 & 519.0725 \\
\hline
\end{tabular}

Table 2. The distance between the terminus and each unit of demand

\begin{tabular}{cccccccccc}
\hline Distance & 0 & 1 & 2 & 3 & 4 & 5 & 6 & 7 & 8 \\
\hline 0 & 0 & 40 & 60 & 75 & 90 & 200 & 100 & 160 & 80 \\
1 & 40 & 0 & 65 & 40 & 100 & 50 & 75 & 110 & 100 \\
2 & 60 & 65 & 0 & 75 & 100 & 100 & 75 & 75 & 75 \\
3 & 75 & 40 & 75 & 0 & 100 & 50 & 90 & 90 & 150 \\
4 & 90 & 100 & 100 & 100 & 0 & 100 & 75 & 75 & 100 \\
5 & 200 & 50 & 100 & 50 & 100 & 0 & 70 & 90 & 75 \\
6 & 100 & 75 & 75 & 90 & 75 & 70 & 0 & 70 & 100 \\
7 & 160 & 110 & 75 & 90 & 75 & 90 & 70 & 0 & 100 \\
8 & 80 & 100 & 75 & 150 & 100 & 75 & 100 & 100 & 0 \\
\hline
\end{tabular}

The results obtained in Table 1 are the results of the overall calling program. Fig. 1 is the result of 50 ants and iterated 100 times when the ACO is called separately under the same parameters. The two results coincide with the whole. The fluctuation of the average shortest path distance is $0.076 \%$. Parameter combination optimization and single call cycle 100 times the shortest distance fluctuation is $1 \%$, it shows that this method of calculation is feasible. 


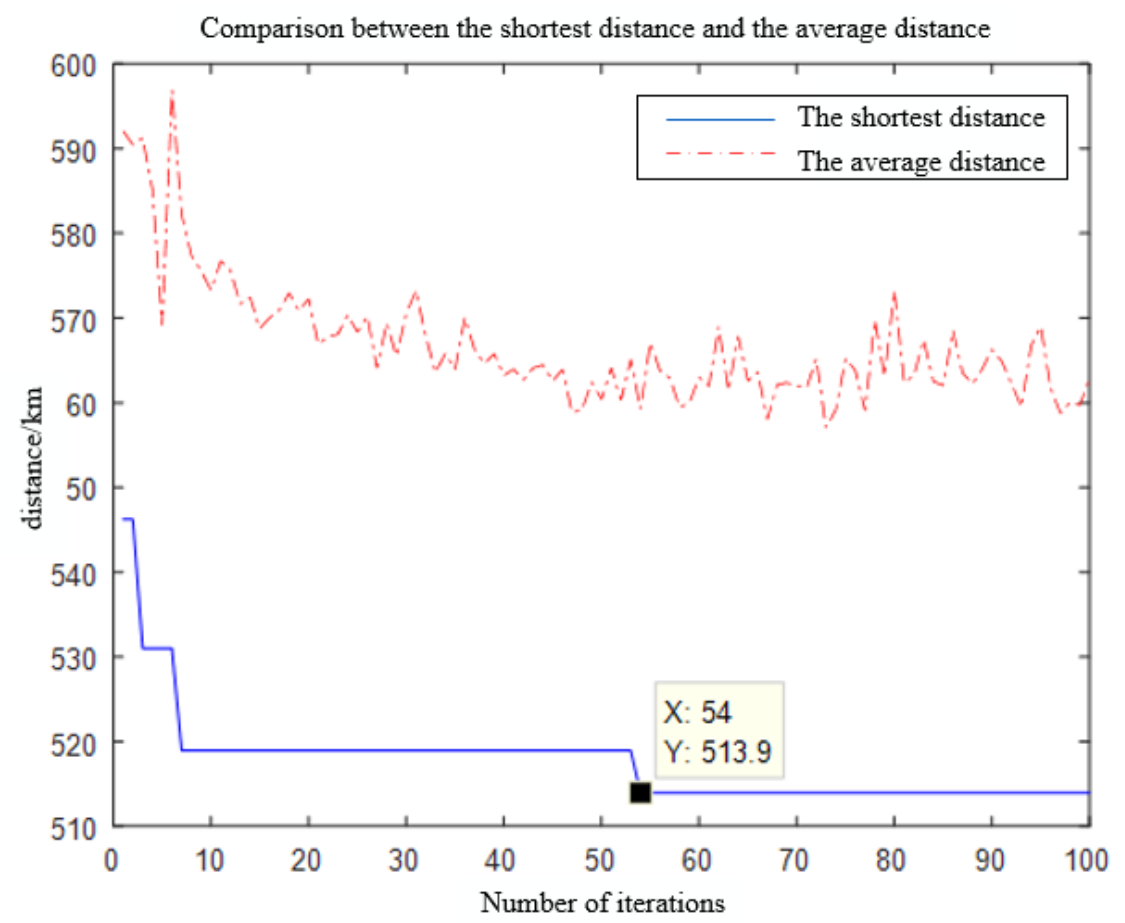

Figure 1. The optimal solution the shortest distance and the optimal distance

\section{Conclusion}

In order to solve the problem that the ant colony algorithm is difficult to determine the optimal combination while selecting the key parameters, a particle swarm optimization algorithm is proposed for adaptive adjustment. Considering the large amount of particle swarm optimization in particle swarm optimization, the reverse learning strategy is introduced to improve the computational efficiency. The randomness of computation is fully considered in the experiment. The average value is calculated by statistics principle. The rationality of the method is proved by calling several iterations repeatedly. However, the theoretical analysis of the algorithm needs to be improved, and the algorithm is random in some parts, and a large number of experiments are needed to support the stability of the experimental results. In the future, we will further improve the research of relevant theoretical work and improve the application of the algorithm in more complex environment.

\section{Acknowledgments}

This work is supported by the National Natural Science Foundation of China under Grant No. 91746116, and Science and Technology Project of Huizhou Province under Grant Nos. [2017]2308, Talents [2015]4011 and [2016]5013, Collaborative Innovation [2015]02.

\section{References}

[1]. Dorito M, Gambardella M. A cooperative learning approach to the traveling salesman problem [J]. 1997.

[2]. Stutz H, Hoops H. Max-min Ant system [J]. Future Generation Computer System, 2000, 6(8):889-914.

[3]. Chai Baoji, Liu Dewey. Application of ant colony algorithm based on Particle Swarm Optimization in TSP [J]. Computer Simulation, 2009, 26(8):89-91.

[4]. Huang Hua, Men Jaunting, Chen Shashti, et al. Study on Vehicle Routing optimization for fruit and vegetable Delivery Based on Improved Ant Colony Algorithm[J]. Storage and Process, 2011, 11(3):24-27. 
[5]. Yang Yana. Parameter optimization of ant colony algorithm and its application [D]. Master Thesis of Nanjing University of Science and Technology, 2008.

[6]. Wang Sherwin, Ding Lexan, Xin Datong, et al. A swarm search optimization algorithm using Reverse learning method [J]. Computer Science, 2012, 39(9):183-187.

[7]. You Xiaoping, Liu Sheng, Lb Jonquil. Ant colony algorithm based on dynamic search strategy and its application on path planning of robot [J]. Control and decision, 2017, 32(3):552-556.

[8]. Wang H, Li H, Liu Y, et al. Opposition-based particle swarm algorithm with Cauchy mutation[C] Evolutionary Computation, 2007. CEC 2007. IEEE Congress on. IEEE, 2007:4750-4756.

[9]. Chen Rue, Zhao Zhuang, Zhang Yanbu, et al. Multi-objective two-sided matching problem based on improved particle swarm ant colony algorithm [J]. Computer engineering and design, 2017, 38(1):220-225.

[10]. Wang $\mathrm{H}, \mathrm{Wu} \mathrm{Z}$, Rahnamayan S, et al. Enhancing particle swarm optimization using generalized opposition-based learning [J]. Information Sciences an International Journal, 2011, 181(20):4699-4714.

[11]. Pu Xingcheng, Li Junkier, Wu Huizhou, et al. Multi objective point path planning of mobile robot based on Improved Particle Swarm Optimization [J]. CAAI Transactions on Intelligent Systems, 2017, 12(3):301-309.

[12]. Xia Hue, Wang Hua, Chen Xi. An ant colony parameter adaptive optimization algorithm based on particle swarm optimization [J]. Journal of Shandong University (Engineering Science), 2010, 40(3):26-30.

[13]. Li Qing, Zhang Chao, Chen Peng, et al. An improved ant colony algorithm based on Parameter Optimization of particle swarm optimization [J]. Control and Decision, 2013(6):873-878.

[14]. Wang Hong Jun, Xu Jun, Zhao Hue, et al. Path planning of robot based on smoothing ant colony algorithm [J]. Journal of Yanshan University, 2017, 41(3):278-2. 